\title{
Determinação da espessura da interface sedimento-embasamento da Bacia Sergipe-Alagoas a partir da assinatura gravimétrica.
}

\author{
Determination of the sediment-basement interface thickness of the Sergipe-Alagoas Basin \\ from the gravimetric data.
}

\author{
DUTRA $^{1}$, A. C.; ARGOLLO ${ }^{2}$, R. M. \\ alannacd@ufba.br;
}

\begin{abstract}
Resumo
Este trabalho utilizou dados gravimétricos para pesquisar o arcabouço da bacia sedimentar Sergipe-Alagoas e parte do embasamento. Os principais objetivos são estimar parâmetros como espessura do embasamento e variação lateral do contraste de densidade nos domínios Estância, Canudos-Vaza-Barris, Macururé e Terreno PernambucoAlagoas no trecho adjacente à Bacia Sergipe-Alagoas, utilizando medidas estruturais geológicas e geofísicas em rochas coletadas na superfície. A partir dos dados adquiridos realizamos o processamento e interpretação de dados gravimétricos ao longo da região do embasamento adjacente e sua extensão para a Bacia Sergipe-Alagoas, para que fosse feita uma modelagem gravimétrica dos domínios crustais e suas relações tectônicas. Para delinear o relevo do embasamento da Bacia Sergipe-Alagoas foi necessário conhecer as anomalias gravimétricas e o contraste de densidade na superfície da bacia. Com estas informações foi possível estimar, além do relevo, o valor do contraste de densidade na superfície desta bacia sedimentar, sendo fornecida a anomalia gravimétrica e a profundidade do embasamento em alguns pontos da bacia. A grande vantagem do método gravimétrico é a capacidade de recobrimento espacial com custos razoáveis, e são bastante utilizados para a detecção, localização e delineação de feições estruturais.
\end{abstract}

Palavras-chave: Bacia-Sedimentar; Dado Gravimétrico; Inversão.

\begin{abstract}
This work used gravimetric data to search the framework of the Sergipe-Alagoas sedimentary basin and part of the basement. The main objectives are to estimate parameters such as basement thickness and lateral variation of density contrast in the Estância, Canudos-Vaza-Barris, Macururé and Pernambuco-Alagoas Terrain areas adjacent to the Sergipe-Alagoas Basin, using geological and geophysical measurements on collected rocks on the surface. From the data acquired, we performed the processing and interpretation of gravimetric data along the region of the adjacent basement and its extension to the SergipeAlagoas Basin, in order to make a gravimetric modeling of the crustal domains and their tectonic relationships. In order to delineate the basement relief of the SergipeAlagoas Basin, it was necessary to know the gravimetric anomalies and the density contrast on the surface of the basin. With this information it was possible to estimate, besides the relief, the density contrast value on the surface of this sedimentary basin, being provided the gravimetric anomaly and depth of the basement in some points of the basin. The great advantages of gravimetric methods are good spatial coating capability with reasonable costs, and are widely used for the detection, localization and delineation of structural features.
\end{abstract}

Keywords: Sedimentar-Basin; Gravimetric Data; Inversion.

\section{INTRODUÇÃO}

A Bacia Sergipe-Alagoas situa-se na margem continental Nordeste do Brasil, sendo limitada pelos paralelos $9^{\circ}$ e $11^{\circ} 30^{\prime} \mathrm{S}$, aproximadamente (Figura 1). Em sua porção terrestre abrange uma área de aproximadamente $13.000 \mathrm{~km}^{2}$ e sua porção submersa uma área de cerca de $40.000 \mathrm{~km}^{2}$, até a cota batimétrica de 2.000 m (Feijó e Vieira, 1991).

\footnotetext{
${ }^{l}$ Alanna Costa Dutra, Departamento de Física da Terra e do Meio Ambiente/Laborarório de Geotermia, Intituto de Física, Salvador-BA, Brasil.

${ }^{2}$ Roberto Max de Argollo, Departamento de Física da Terra e do Meio Ambiente/Laborarório de Geotermia, Intituto de Física, 


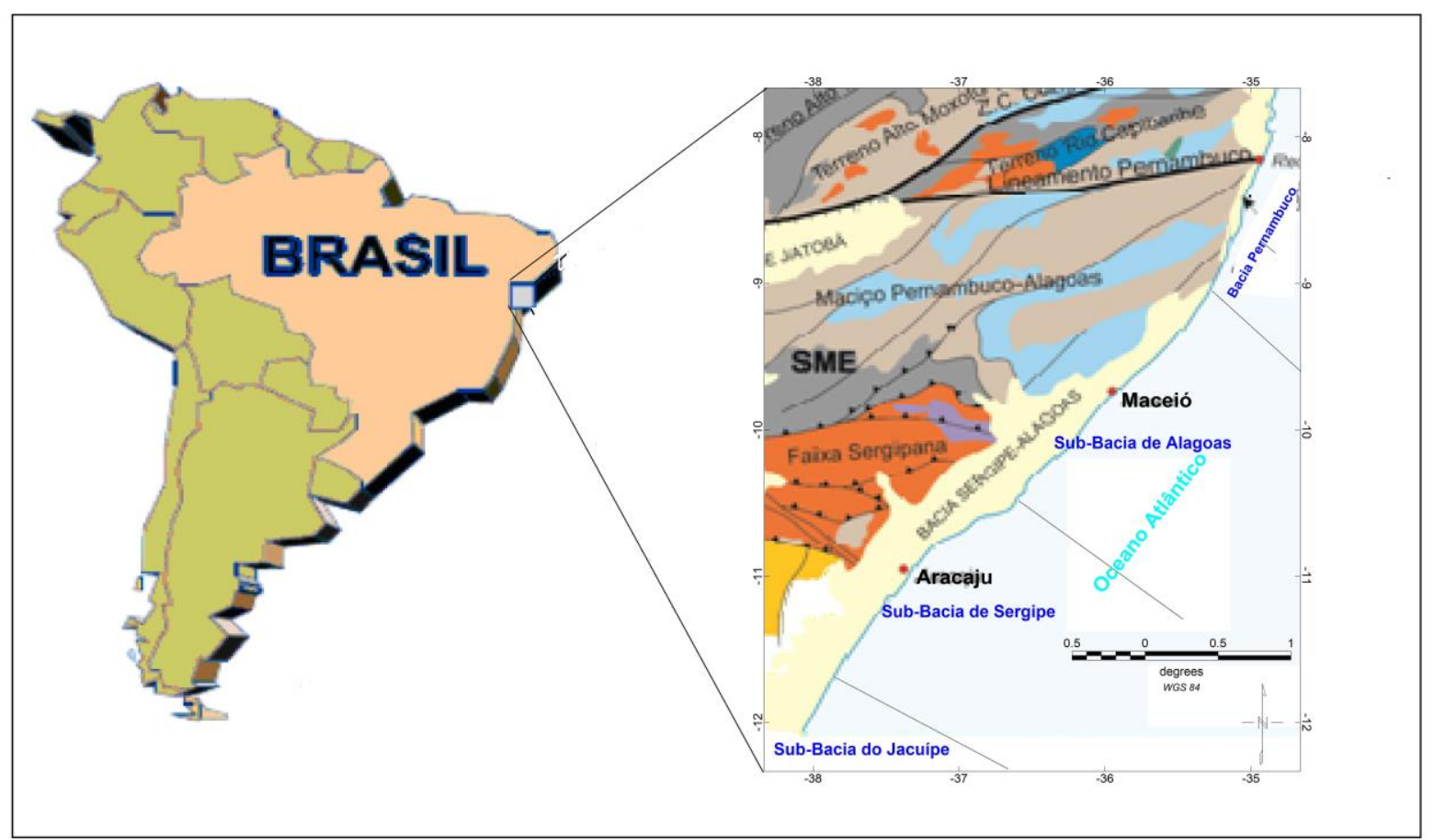

Figura 1. Mapa de localização da Bacia Sergipe-Alagoas e divisão geotectônica da Sub- Província Borborema Meridional (SME) de acordo com Santos et al., (2014). Fonte: Dutra e Argollo (2017).

A bacia é subdividida em dois compartimentos, um ao norte, denominado Sub-bacia de Alagoas (SBAL) e outro a sul, denominado Sub-bacia Sergipe (SBSE), separados, grosso modo, pelos Altos de Japoatã (SE) e Penedo (AL) (Cruz 2008).

A Bacia Sergipe-Alagoas está delimitada ao sul pela Plataforma Estância, que se separa da Bacia de Jacuípe e, a norte, pelo Alto de Maragogi, que a separa da Bacia Pernambuco-Paraíba. O embasamento cristalino a oeste da bacia é composto por duas unidades geotectônicas distintas: A Faixa Sergipana, na porção sul, e o Terreno Pernambuco-Alagoas, na porção norte (Cruz 2008) (Figura 2).

Esse embasamento compreende os domínios geotectônicos Salvador-Itabuna-Curaçá da subprovíncia cráton do São Francisco, e Estância, Canudos-Vaza-Barris, Macururé, Rio Coruripe e Pernambuco- Alagoas da sub-província Meridional da província Borborema, os quais devem constituir o embasamento da Bacia Sergipe-Alagoas (Brito Neves et al., 2000, Oliveira et al., 2010; Santos et al., 2000).

De todas as bacias da margem continental brasileira, esta é a que registra a sucessão estratigráfica mais completa, incluindo remanescentes de uma sedimentação paleozóica, um pacote jurássico a eocretácico pré-rifte amplamente desenvolvido e as clássicas seqüências mesocenozóicas sinrifte e pós-rifte (Cainelli; Mohriak, 1998). A bacia é representada por um rifte 
assimétrico, alongado na direção NNE/SSW e limitado a norte com a Bacia Pernambuco-Paraíba, pelo Alto de Maragogi, e a sul com a Bacia do Jacuípe, pelo sistema de falhas de Vaza-Barris e granitóides do Arqueano.

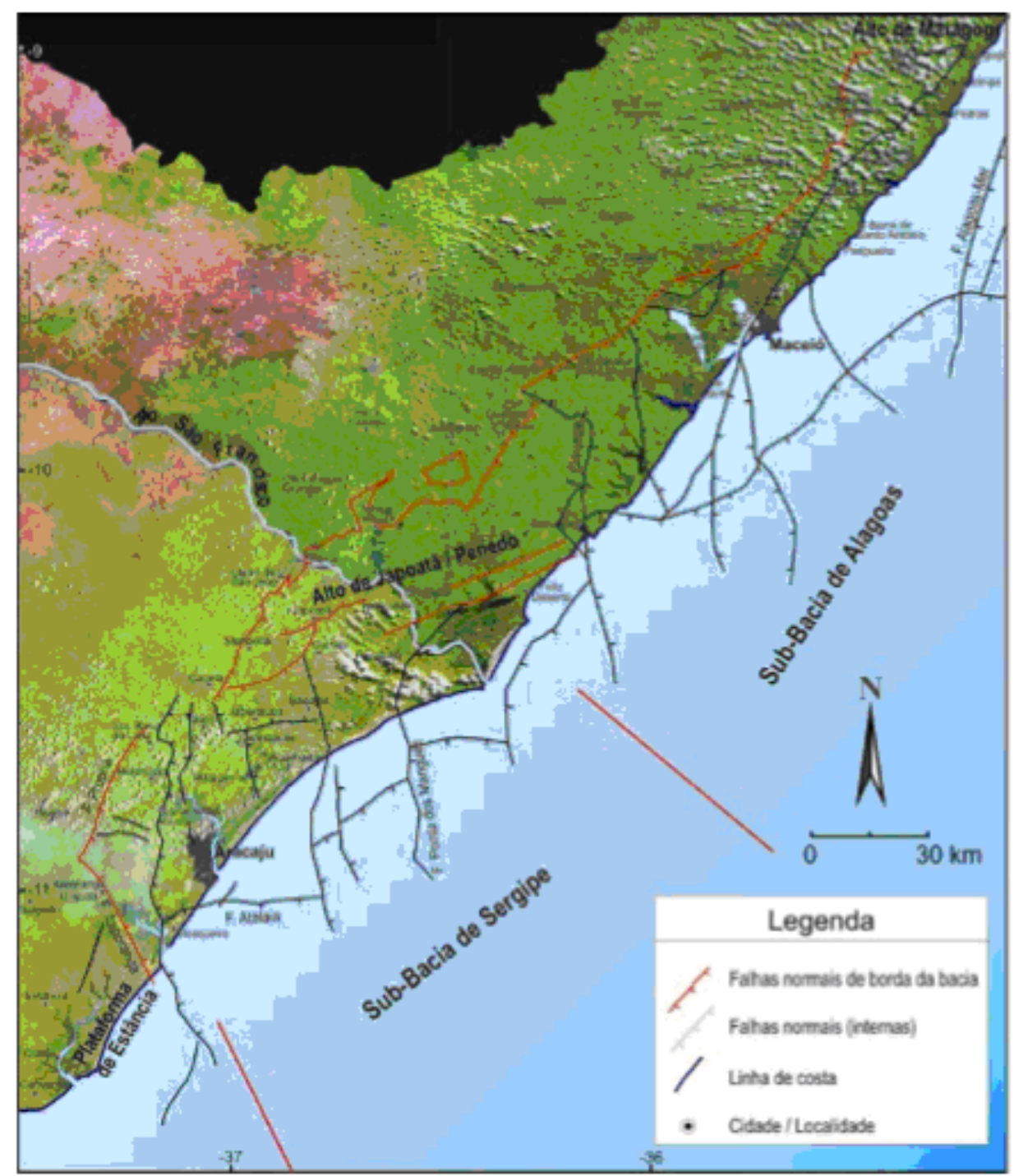

Figura 2. Mapa da Bacia Sergipe - Alagoas retirado de Cruz (2008) mostrando a subdivisão nas Sub-bacias de Sergipe, ao sul, e Alagoas, ao norte. O arcabouço estrutural e os compartimentos tectônicos foras definidos por Falkenhein et al (1986). Fonte: Cruz (2008).

As duas sub-bacias, Alagoas e Sergipe, são separadas pelo alto de Jaboatã-Penedo, nas imediações do Rio São Francisco (Aquino e Lana, 1990). A Bacia de Sergipe-Alagoas é caracterizada por uma espessa seção sedimentar da fase rifte na região continental e por feições associadas à transpressão na região da plataforma e talude. Entre as sub-bacias de Alagoas e Sergipe ocorrem feições diapíricas em águas profundas que podem estar relacionadas à tectônica de sal (Mohriak, 1995). Em uma bacia, o calor radiogênico gerado pelas rochas do embasamento e pelas camadas sedimentares, somado ao calor proveniente da astenosfera, resulta no fluxo de calor superficial total. Esse fluxo desempenha um papel relevante na história térmica da bacia, sendo seu 
conhecimento importante para avaliar a geração de hidrocarbonetos. Essa avaliação, realizada por meio da modelagem termomecânica da bacia, requer o conhecimento da massa específica e das propriedades térmicas das rochas do embasamento e sedimentos das bacias, além da geometria e profundidade das fontes (Mottaghy et. al, 2005).

Foram utilizados dados gravimétricos para pesquisar o arcabouço da bacia sedimentar Sergipe-Alagoas e parte do embasamento (Figura 2). Os principais objetivos são estimar parâmetros como espessura do embasamento e variação lateral do contraste de densidade nos domínios Estância, Canudos-Vaza-Barris e Macururé e Terreno Pernambuco-Alagoas no trecho adjacente à Bacia Sergipe-Alagoas, utilizando medidas geológicas e geofísicas em rochas coletadas na superfície.

Este trabalho insere-se no estudo mais geral desenvolvido no projeto Geoterm-Ne "Geração de calor na bacia Sergipe-Alagoas no embasamento a ela adjacente" do Programa Tecnológico de Exploração de Modelagem de Bacias (Promob) do Centro de Pesquisas da Petrobras (Cenpes), executado no Centro de Pesquisa em Geofísica e Geologia da Universidade Federal da Bahia (CPGG/UFBA).

\section{METODOLOGIA}

Neste projeto foram utilizados os dados gravimétricos obtidos em campanhas (2014/2015), além de dados disponíveis na Rede Nacional de Gravimetria (Figura 3). Realizamos transeção gravimétrica em até $300 \mathrm{~km}$ de linha, cobrindo áreas de interesse. Para esta transeção, contamos com o levantamento planialtimétrico necessário com GPS diferencial e as medidas gravimétricas com um gravímetro Scintrex CG-5.

Após a aquisição de dados gravimétricos segue-se com a etapa de processamento, conhecida como redução, que transforma as leituras do equipamento em acelerações e anomalias de gravidade para que sejam interpretadas na forma de mapas e perfis.

A aquisição, processamento e interpretação de dados gravimétricos foram realizados para obtenção de soluções de profundidade do embasamento da Bacia Sergipe-Alagoas, conhecendo as anomalias gravimétricas, o contraste de densidade na superfície da bacia (Last e Kubic, 1993; Barbosa e Silva, 1994; Barbosa et al, 1997; Barbosa et al, 1999). Utiliza-se primeiramente a formulação linear como formulação do problema inverso, cujo substrato foi representado por uma malha homogênea, de posição e tamanho conhecidos, mas com contraste da propriedade física 
desconhecido. A inversão de dados consistiu em determinar a propriedade física em cada célula e, com isto, delinear a geometria da fonte verdadeira.

Um segundo método utilizou a formulação não-linear no qual as fontes são representadas por prismas justapostos, com contraste da propriedade física conhecido, mas com a base da bacia a ser determinada. Nós utilizamos um método estável para interpretar a relevo do embasamento da bacia sedimentar Sergipe-Alagoas. Este método que delineia descontinuidades no relevo do embasamento.

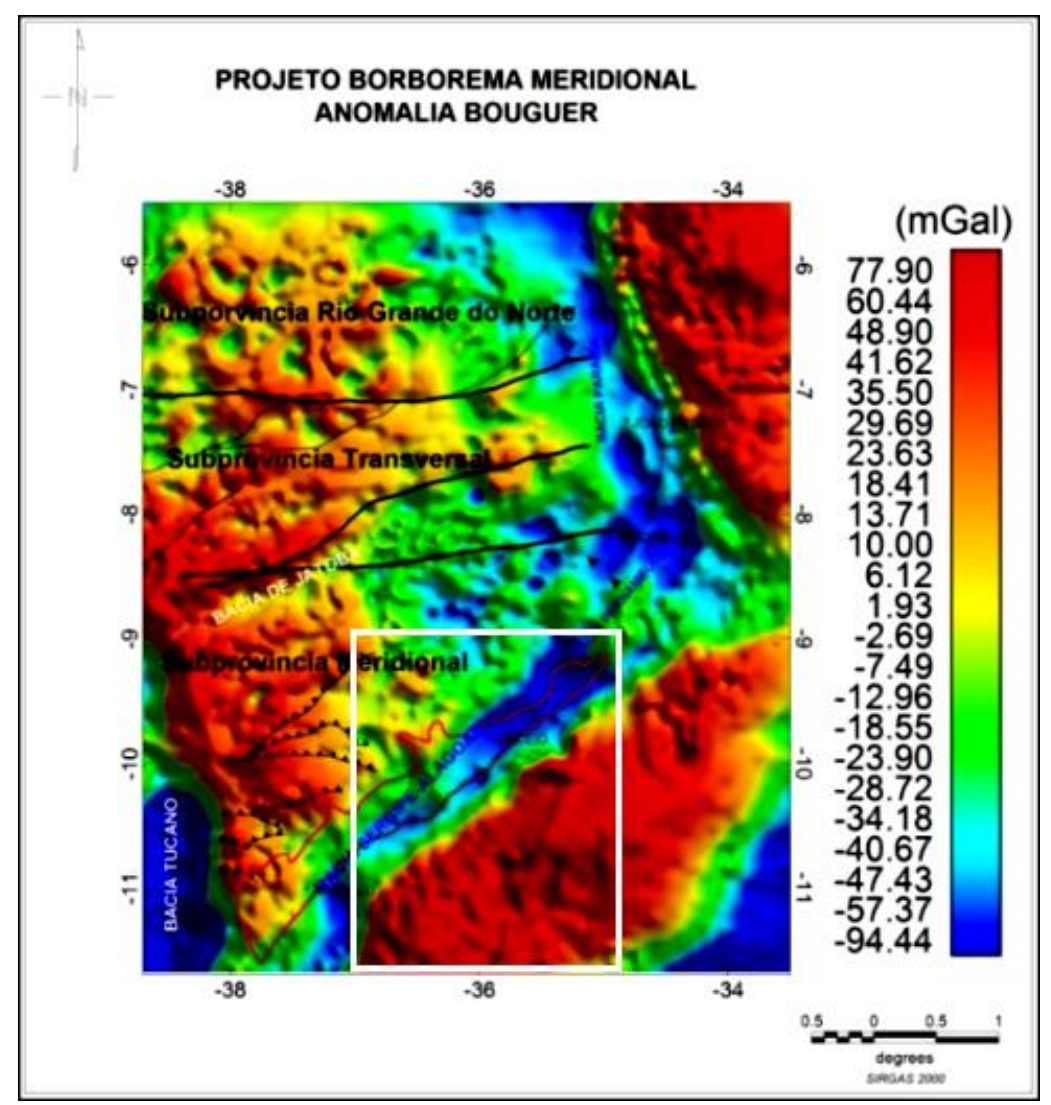

Figura 3. Mapa tectônico esquemático da Província Borborema (Oliveira, 2008), mostrando anomalias gravimétricas e alinhamentos de zonas de fraturas continentais. A linha vermelha representa o limite geológico da Bacia Sergipe-Alagoas e a linha em branco representa a área de estudo. Fonte: Dutra e Argollo (2017).

\subsection{GRAVIMETRIA}

A gravidade varia de ponto a ponto sobre toda a superfície terrestre. As variações com causas diversas se superpõem e se confundem mutuamente. Quando o objetivo é o de se estudar variação da gravidade causada por diferenças de densidade na crosta e no manto é necessário eliminar todas as demais variações conhecidas (variações causadas por: rotação e o achatamento da Terra, atração da Lua e do Sol, variações de altitude entre os pontos de medida, efeitos da estrutura da crosta e manto superior). O processo de eliminação dessas variações é chamado de "correção 
gravimétrica" e os valores resultantes são conhecidos como "anomalias gravimétricas". As equações usadas nesse procedimento são encontradas em textos didáticos como Lowrie (1997) e Blakely (1996).

A anomalia gravimétrica deriva da presença de diferentes densidades na Terra, sendo que o método gravimétrico é sensível quando essas diferenças se encontram lado a lado. A maioria das rochas tem densidades entre 1,6 e 3,2 $\mathrm{g} / \mathrm{cm}^{3}$. A densidade depende da composição e da porosidade. As rochas sedimentares, na média, apresentam valores mais baixos, em função da porosidade, e da densidade do fluido que preenche os poros. Por isso, as sequências de rochas sedimentares apresentam normalmente um aumento da densidade em função da profundidade devido à compactação. Valores de densidade constantes para o pacote sedimentar inicialmente presumido podem ser posteriormente substituídos por modelos interpretativos que admitem um aumento da densidade dos sedimentos com a profundidade. Por exemplo, Cordell (1973) e Chai e Hinze (1988) presumem que o contraste de densidade decai de forma exponencial. Murthy e Rao (1979) utilizaram a equação da anomalia de um modelo poligonal cujo contraste de densidade varia linearmente com a profundidade. Bhaskara Rao (1986) estabeleceu uma variação quadrática para o contraste de densidade e Sari e Şalk (2002) utilizam um contraste de densidade variando hiperbolicamente com a profundidade, introduzido por Litinsky (1989) para delinear o embasamento de bacias sedimentares.

A aparência da anomalia depende das dimensões, contraste de densidade e profundidade do corpo anômalo. A extensão horizontal da anomalia é chamada de comprimento de onda aparente. Este é uma medida da profundidade do corpo. Corpos profundos tendem a produzir anomalias mais achatadas que ocupam uma área maior (longo comprimento de onda). Corpos mais rasos tendem a produzir anomalias estreitas e de amplitude mais definida (pequeno comprimento de onda). As anomalias de grande comprimento de onda, de origem profunda tendem a ser chamadas de regional, enquanto que as anomalias de pequeno comprimento de onda são chamadas de residual.

\subsection{MODELO GRAVIMÉTRICO E INVERSÃO DE DADOS}

Para o campo magnético anômalo $\mathrm{g}\left(\mathrm{x}_{\mathrm{i}}, \mathrm{y}_{\mathrm{i}}, \mathrm{z}_{\mathrm{i}}\right)$ produzido pela distribuição de densidade $\left(\mathrm{p}_{\mathrm{j}}\right)$ tem-se a seguinte equação:

$$
g_{i}=\gamma \sum_{j} d_{j} \int_{x o_{j}-a}^{x o_{j}} \int_{y o_{j}-a}^{+a} \int_{0}^{a o_{j}+a} \frac{z-z^{\prime}}{\left[\left(x_{i}-x_{j}\right)^{2}+\left(y_{c}-y_{j}^{\prime}\right)^{2}+\left(z_{i}-z^{\prime}{ }_{j}\right)^{2}\right] \frac{3}{2}} d x_{j}^{\prime} d y_{j}^{\prime} d z_{j}^{\prime}
$$


Em que $\gamma$ constante gravitacional universal e $g_{i}$ é o campo gravimétrico produzido pelo jésimo prisma retangular. As observações $g_{i}\left(x_{i}, y_{i}, z_{i}\right)$ são aproximadas por um funcional contínuo expressando a relação entre a propriedade física e as observações magnéticas correspondentes.

Assim temos um conjunto de observações geofísicas produzidas por fontes arbitrárias, adotando-se o sistema de coordenadas cartesianas. Geralmente assume-se que as fontes são corpos bi ou tridimensionais, dependendo do algorítimo utilizado. Inclui-se a fonte em uma região que represente o substrato e que é discretizada em M células. Dentro de cada célula assume-se que a propriedade física seja constante. As observações são aproximadas por um funcional contínuo expressando a relação entre a propriedade física e as observações geofísicas correspondentes; temos um conjunto de parâmetros associados a geometria e a posição de cada célula e p é um vetor dimensão $\mathrm{M}$ das propriedades física.

Em alguns métodos utilizam-se a formulação linear como formulação do problema inverso cujo substrato é representado por uma malha homogênea, de posição e tamanho conhecidos, mas com contraste da propriedade física desconhecido, conforme ilustrado na Figura 4. A inversão de dados consiste em determinar a propriedade física em cada célula e, com isto, delinear a geometria da fonte verdadeira (Guillen; Menichetti, 1984; Last; Kubic, 1993; Barbosa; Silva, 1994; Long; Kaufmann, 2013; Hinze et al., 2013).

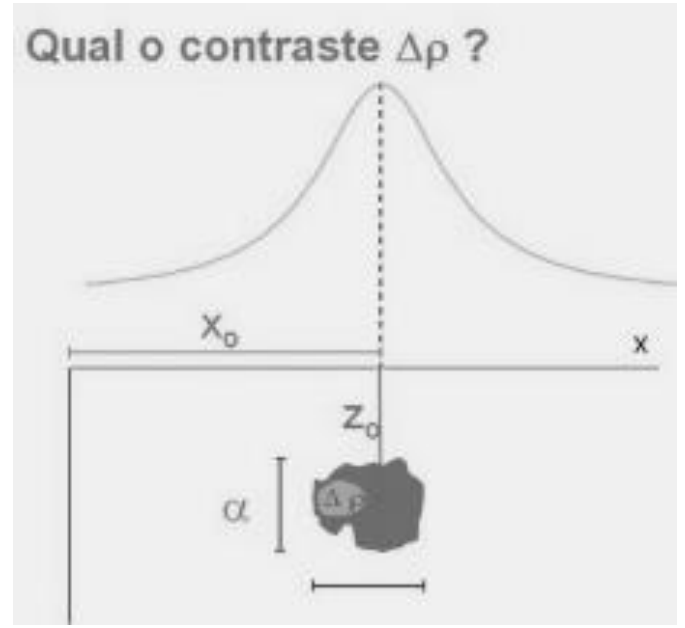

Figura 4. Esquema mostrando a anomalia causada pelo contraste de densidade entre o corpo anômalo e o entorno. Fonte: Dutra e Argollo (2017).

Outros métodos utilizam a formulação não-linear no qual as fontes são representadas por prismas justapostos, com contraste da propriedade física conhecido, mas com topo ou a base a serem determinados (Barbosa et al, 1997; Barbosa et al, 1999). 


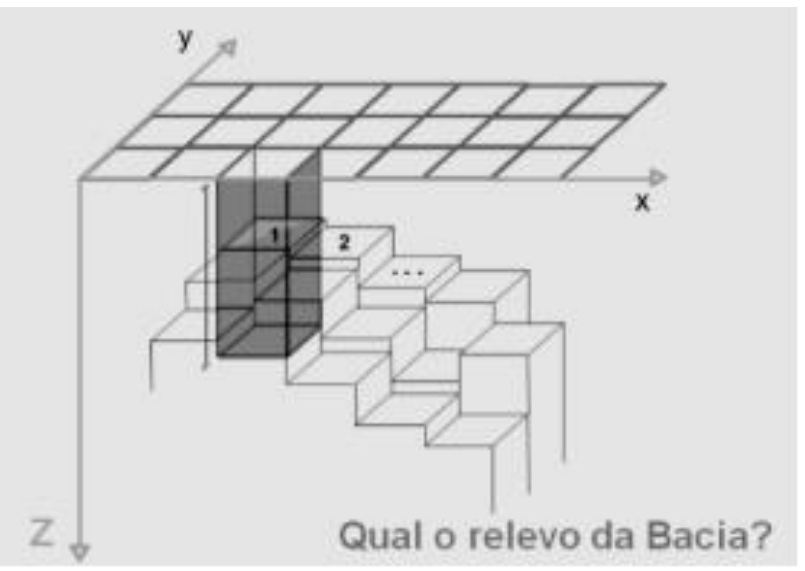

Figura 5. Esquema mostrando a região em subsuperfície contendo a bacia discretizada em uma grade de prismas elementares justapostos, cujas espessuras $(p)$ são parâmetros a serem estimados. Fonte: Dutra e Argollo (2017).

O modelo interpretativo consiste de uma grade de prismas retangulares verticais justapostos. Por simplificação, os centros dos prismas coincidem com os pontos de observação. Os parâmetros a serem determinados são as espessuras $p_{j}$ dos prismas, conforme ilustrado na Figura 5.

O ajuste dos dados é imposto pela minimização da função:

$$
\phi^{g}\left(\mathbf{g}, \mathbf{g}^{0}\right)=\frac{1}{N}\left\|\mathbf{g}(\mathbf{p})-\mathbf{g}^{0}\right\|^{2}
$$

estimar $p$ de $g^{0}$ é um problema inverso não linear. É necessário incorporar um vínculo de suavidade, introduzindo uma ponderação no grau de suavidade entre as estimativas de parâmetros (nesse caso espessuras) contíguos. Isso é conseguido através da minimização do funcional

$$
\phi^{s}(\mathbf{p})=\mathbf{p}^{\mathrm{T}} \mathbf{R}^{\mathrm{T}} \mathbf{W}^{\mathrm{T}} \mathbf{W} \mathbf{R} \mathbf{p}
$$

em que $f^{\mathrm{r}}$ é o fator normalizado, $\mathbf{R}$ é uma matriz cujas linhas contém apenas dois elementos não nulos iguais a 1 e -1 , localizados nas colunas correspondentes aos parâmetros i e j cujas estimativas devem estar o mais próximas possível. Estes parâmetros estão associados com cada para de parâmetros adjacentes (Medeiros and Silva, 1996). K é o número total de pares de parâmetros adjacentes. W é uma matriz diagonal de pesos usada para relaxar o grau de suavidade imposto a determinados pares de parâmetros. Este tipo de vínculo é denominado Suavidade Ponderada. Maiores detalhes podem ser obtidos em Barbosa et al. (1999). Na k-ésima iteração, o i-ésimo elemento diagonal da matriz $\mathrm{W}$ é dado essencialmente por: 


$$
e_{i i(k)}=\exp \left[-b\left(\hat{p}_{j(k)}-\hat{p}_{l(k)}\right)\right]
$$

em que os subscritos $j$ e $l$ definem o i-ésimo par de parâmetros adjacentes e b é um fator que controla o quanto o desnível $\left(\hat{p}_{j(k)}-\hat{p}_{(k)}\right)$ - deverá ser acentuado na iteração k+1.

O papel da matriz $\mathbf{W}$ é permitir um aumento de resolução (em relação ao vínculo de suavidade tradicional) na estimação de relevos descontínuos. Essa tentativa de aumento de resolução, entretanto, leva a um inevitável decréscimo na estabilidade das soluções.

Para compensar essa perda de estabilidade, mais informação a priori deve ser introduzida. Essa informação será que o fundo da bacia é virtualmente plano e localizado a uma profundidade conhecida. Essa informação é imposta através da incorporação de dois vínculos adicionais: a desigualdade e a igualdade absoluta, ou seja, proximidade a alguns valores absolutos. Assim, primeiramente impõe-se que os parâmetros devem satisfazer à desigualdade

$$
0<\mathbf{p}<\mathbf{p}^{\text {sup }},
$$

em que 0 é o vetor nulo, $\mathrm{p}^{\text {sup }}$ é o vetor cujos elementos são iguais a um limite $\mathrm{h}_{\text {max }}$, considerado constante. As desigualdades acima são definidas elemento por elemento.

A seguir, impõe-se que todos os parâmetros devem estar o mais próximo possível de um valor a que é igual ou maior que $\mathrm{h}_{\max }$. Como os vínculos de desigualdade são rígidos e os de igualdade absoluta são aproximados, nenhum parâmetro excede $\mathrm{h}_{\max }$. Barbosa et al. (1999).

O vínculo de igualdade absoluta é incorporado através da minimização do funcional:

$$
\phi^{r}=\sum_{i=1}^{M}\left(p_{i}-a\right)^{2}
$$

em que $a$ é um valor atribuído ao limite máximo $(\mathrm{em} \mathrm{km}$ ) das espessuras da bacia sedimentar (um limite $\mathrm{h}_{\max }$ ). Por simplificação, foi estabelecido apenas um único valor a para toda a bacia sedimentar. Assim, os vínculos de suavidade ponderada e igualdade absoluta são incorporados através da minimização do funcional:

$$
\Phi=\sum_{i=1}^{N}\left[y_{i}^{o}-g_{i}(p)\right]^{2}+\mu_{r} \sum_{i=1}^{M}\left(p_{i}-a\right)^{2}+\mu_{s} \mathbf{p}^{\mathrm{T}} \mathbf{R}^{\mathrm{T}} \mathbf{W}^{\mathrm{T}} \mathbf{W} \mathbf{R} \mathbf{p}
$$


em que $\mu_{\mathrm{r}}$ e $\mu_{\mathrm{s}}$ são escalares não negativos conhecidos como multiplicadores de Lagrange. Detalhes do algoritmo podem ser obtidos em Barbosa et al. (1999).

\section{RESULTADOS E DISCUSSÃO}

Foi caracterizado o relevo do embasamento da Bacia Sedimentar Sergipe-Alagoas mapeada gravimetricamente. Os modelos obtidos permitem explicar a distribuição do contraste de densidade no embasamento adjacente à Bacia Sedimentar Sergipe-Alagoas, assim como delineamente de estruturas ou blocos crustais.

O estudo da estrutura de bacias é uma importante aplicação econômica, principalmente em exploração de petróleo e gás. A maior parte do preenchimento da bacia normalmente tem um contraste de densidade muito menor do que o embasamento cristalino. Assim, é geralmente possível estimar a profundidade de embasamento em circunstâncias favoráveis, quantitativamente mapear estruturas, como falhas e blocos.

Visto que tais estruturas em seções rasas residem muitas vezes sobre o embasamento, pelo menos em alguma profundidade, e as falhas em seções rasas são muitas vezes controladas pela reativação de falhas do embasamento, sendo possível identificar estruturas favoráveis ao acúmulo de hidrocarbonetos a partir da interpretação do embasamento.

$\mathrm{Na}$ exploração de hidrocarbonetos, tais técnicas podem ser usadas para ajudar a correlacionar os sistemas complexos para a exploração de falhas ou para reservatório de desenvolvimento. Em áreas onde as camadas carregam uma assinatura gravimétrica e mergulham em um ângulo significativo, uma investigação detalhada pode ser usada para mapear a geologia de superfície de forma muito precisa.

As áreas 1 e 2 (Figura 6) foram definidas a partir da análise dos valores de anomalias mais negativos que representam a maior parte do preenchiemento da bacia que tem um contraste de densidade muito menor do que o embasamento cristalino. Assim foi possível estimar a profundidade do embasamento, bem como mapear estruturas e blocos.

Utilizamos valores de densidade (Figura 7) como referência para rochas da superfície. Esses valores foram utilizados no processo de inversão como informação a priori para obtenção de soluções de contraste de densidade em profundidade das rochas em subsuperfície, conhecendo as anomalias gravimétricas (Figura 8). 


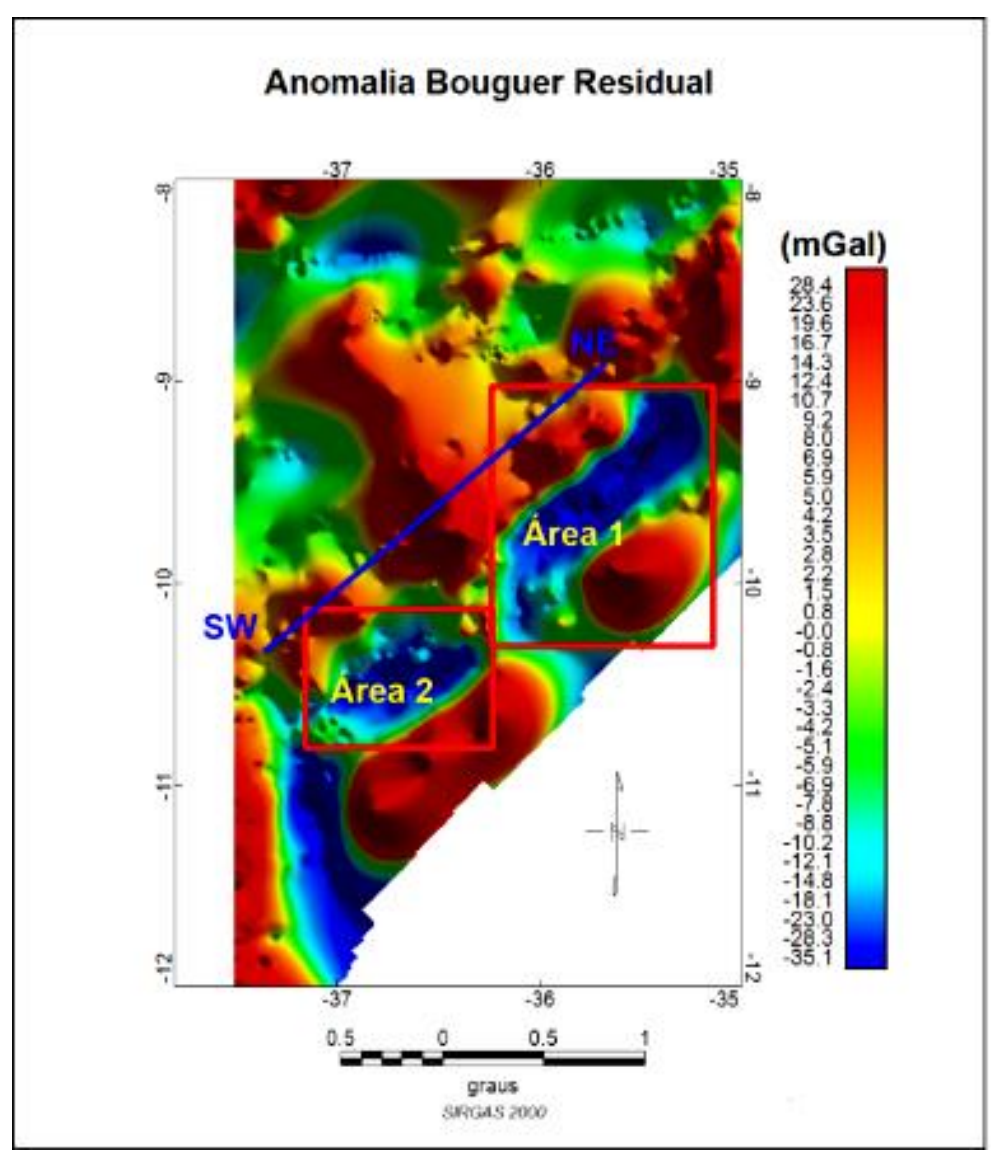

Figura 6. Mapa de anomalia Bouguer Residual e perfil SW-SE utilizado para inversão 2D e obtenção de soluções de contraste de densidade em profundidade. Áreas 1 e 2 para obtenção de soluções 3D para o embasamento. Fonte: Dutra e Argollo (2017).

Nós primeiramente utilizamos a formulação linear como formulação do problema inverso, cujo substrato foi representado por uma malha homogênea, de posição e tamanho conhecidos, mas com contraste de densidade desconhecido (Figura 8). A inversão de dados consistiu em determinar o contraste de densidade em cada célula e, com isto, delinear a geometria da fonte verdadeira.

Interpretações que envolvem a posição e o delineamento dessas fontes compreenderam o método de inversão que determinou automaticamente a posição e a geometria dos corpos. Informação a priori sobre cada fonte, assim como as informações geológicas foram traduzidas matematicamente e incorporadas automaticamente pelo método.

Na Figura 8 apresentamos um modelo de contraste de densidade em profundidade obtido a partir de dados gravimétricos residuais onde cada solução obtida possui um valor máximo de $\Delta \rho=0,25 \mathrm{~g} / \mathrm{cm}^{3}$. As principais estruturas regionais foram mapeadas em profundidade: Zona de Cisalhamento Belo Monte-Jeremoabo, Terreno Pernambuco-Alagoas e DRC = Domínio Rio Coruripe. 


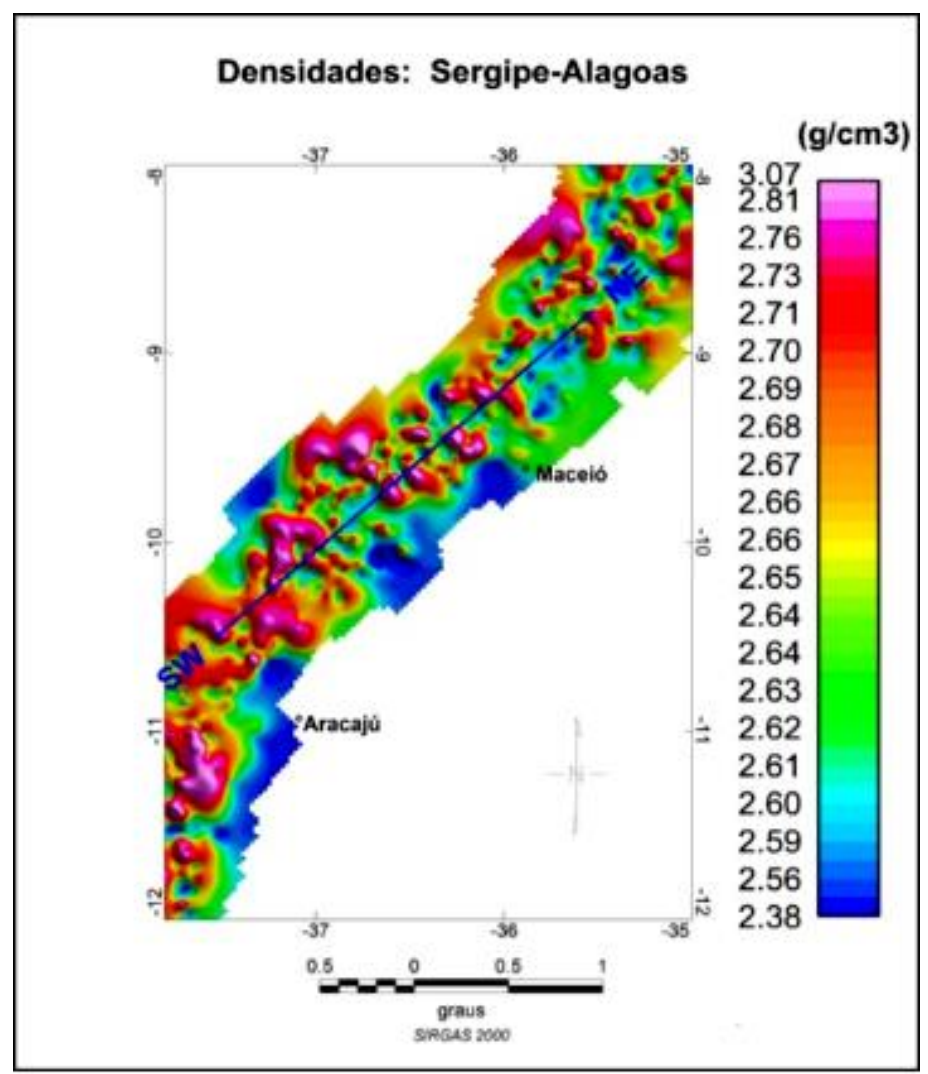

Figura 7. Mapa de distribuição de densidade na superfície adjacente à Bacia Sedimentar Sergipe-Alagoas. Fonte: Dutra e Argollo (2017).
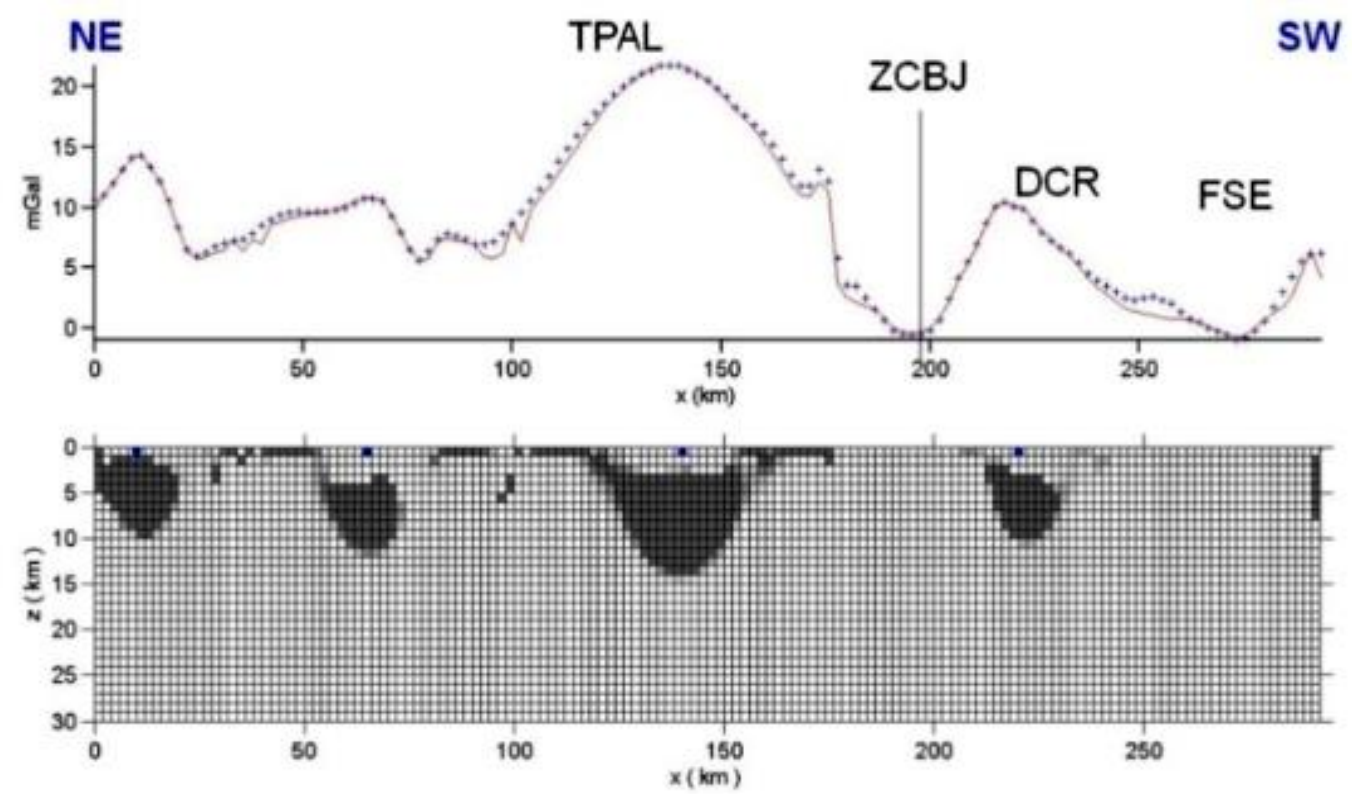

Figura 8. Soluções de contraste de densidade obtidas a partir da inversão de dados gravimétricos. Principais estruturas regionais: $\mathrm{ZCBJ}=$ Zona de Cisalhamento Belo Monte-Jeremoabo; TPAL $=$ Terreno Pernambuco-Alagoas; DRC = Domínio Rio Coruripe; FSE = Faixa Sergipana. Fonte: Dutra e Argollo (2017). 
Com base no exposto, sugere-se que a intrusão dos granitóides da unidade Ortognaisse Serra das Flores variando de 2 a $15 \mathrm{~km}$, essa intrusão ocorreu TPAL o qual atuou de forma significativa nesta porção da Província Borborema, responsável pelo deslocamento para norte das massas de rochas do domínio Rio Coruripe sobre os metamorfitos e plutonitos do Domínio PernambucoAlagoas.

Conhecendo-se a anomalia gravimétrica e o contraste de densidade na superfície da bacia em superfície, foi possível delinear o relevo do embasamento Bacia Sergipe-Alagoas.

Foi utilizada a formulação não-linear para delinear o relevo do embasamento no qual as fontes são representadas por prismas justapostos, com contraste de densidade conhecido, mas com a base da bacia a ser determinada. A parte do trabalho de obtenção de soluções 3D para o embasamento produziu excelentes estimativas do relevo da bacia sedimentar como pode ser visto nas Figuras 9 e 10.

A área 1 contém 1702 estações gravimétricas onde os valores máximos obtidos da espessura foram 4,0 km de profundidade (Figura 9). A área 2 contém 1665 estações gravimétricas onde os valores máximos obtidos da espessura foram 3,50 km de profundidade (Figura 10). Para inverter os dados reais utilizou-se um contraste de densidade de $\Delta \rho=-0,25 \mathrm{~g} / \mathrm{cm}^{3}$.

O método utilizado permitiu o delineamento das descontinuidades no relevo do embasamento. A região em subsuperfície contendo a bacia foi discretizada em uma grade de prismas elementares justapostos, cujas espessuras estimadas representam a profundidade da interface de separação entre os sedimentos e o embasamento da Bacia Sergipe-Alagoas que foi divida em duas áreas (1 e 2). 

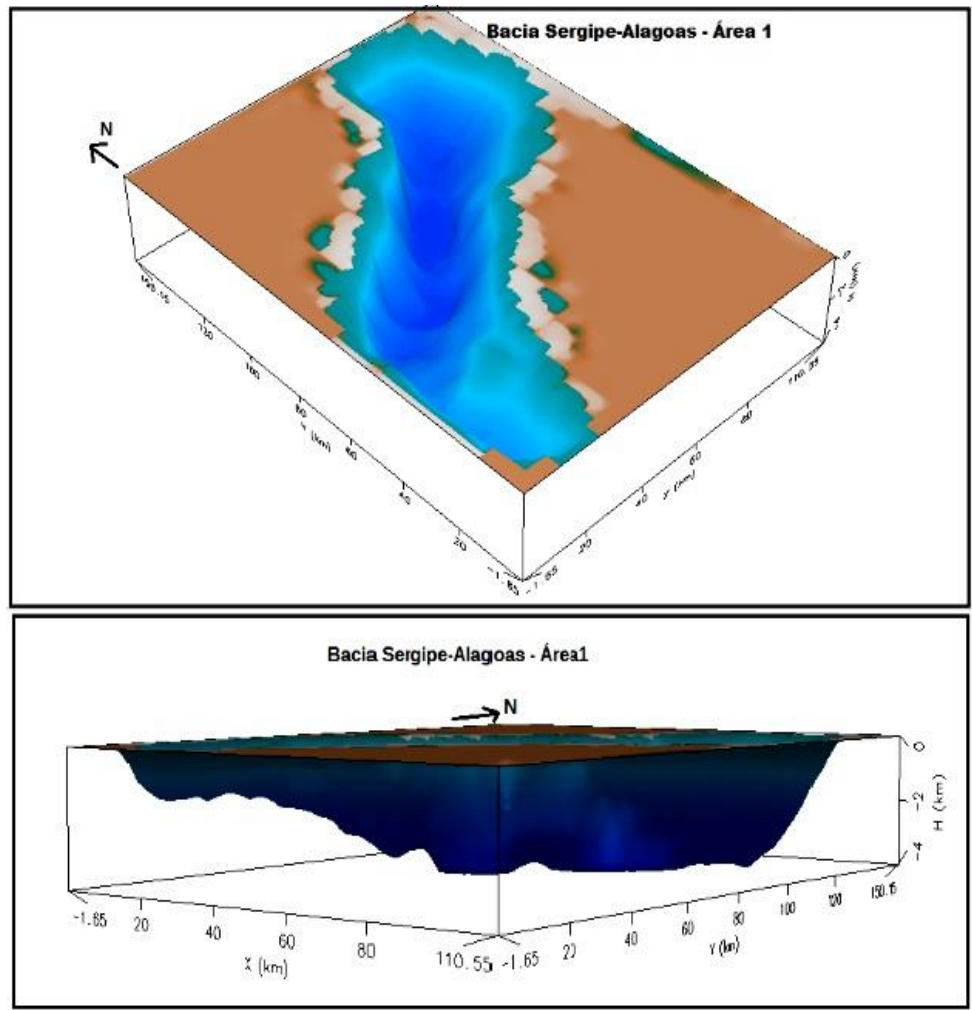

Figura 9. Soluções de espessuras estimadas $h_{j}$ que representam a profundidade da interface de separação entre os sedimentos e o embasamento para Área 1. Fonte: Dutra e Argollo (2017).

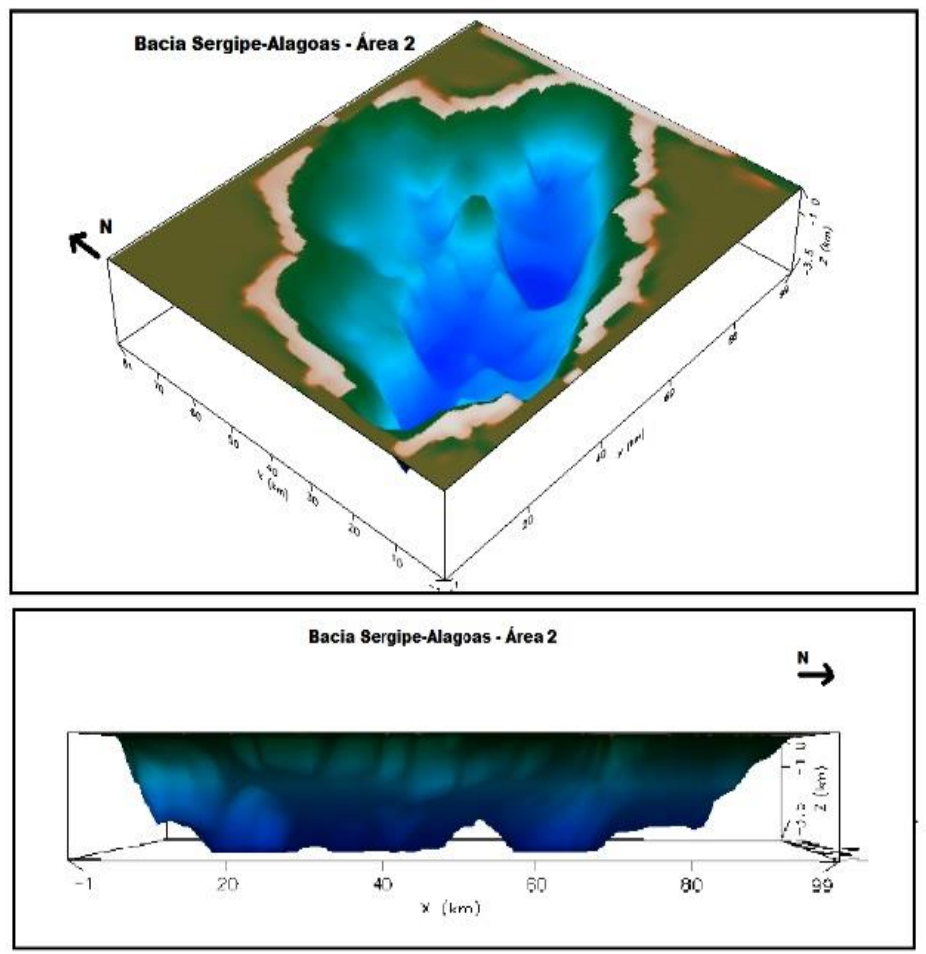

Figura 10. Soluções de espessuras estimadas $h_{j}$ que representam a profundidade da interface de separação entre os sedimentos e o embasamento para Área 2. Fonte: Dutra e Argollo (2017) 
A inversão utilizou como critério de parada o erro do dado menor que $0,1 \%$. Os modelos de profundidades nas inversões apresentam correspondência com as anomalias observadas (Figuras 11 e 12)

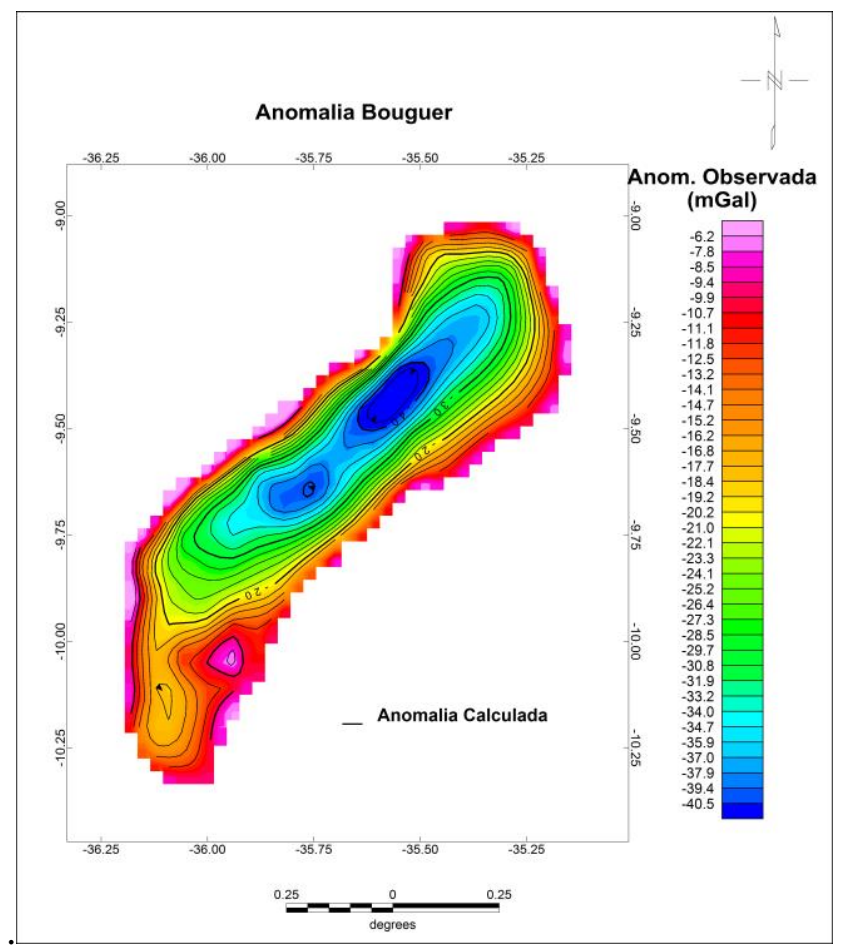

Figura 11. Área 1 - Anomalia Bouguer Residual (mapa em cores) obtidas pela remoção de uma superfície polinomial de ordem três, e anomalias ajustada (linhas contínuas pretas) usando inversão. Intervalo de Contorno de 2.5 mGal. Fonte: Dutra e Argollo (2017)

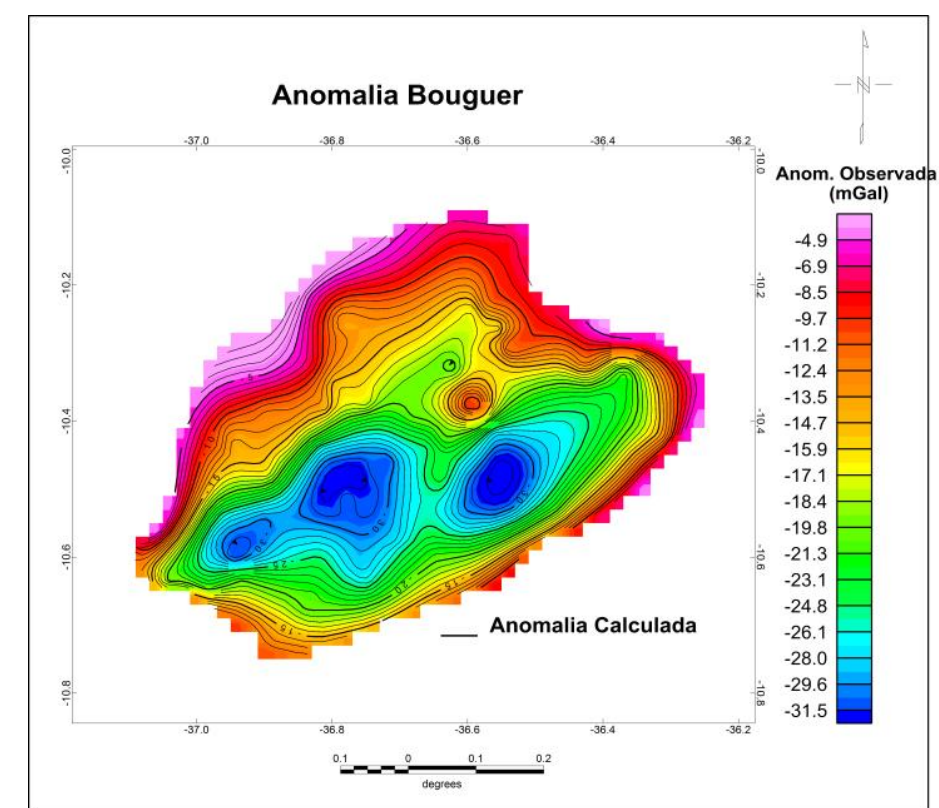

Figura 12. Área 2 - Anomalia Bouguer Residual (mapa em cores) obtidas pela remoção de uma superfície polinomial de ordem três, e anomalias ajustada (linhas contínuas pretas) usando inversão. Intervalo de Contorno de 1.0 mGal. Fonte: Dutra e Argollo (2017). 
A modelagem se mostrou eficiente e o método de inversão também se mostrou eficaz para se obter o delineamento do relevo do embasamento adotando o contraste de densidade de $\Delta \rho=-0,25$ $\mathrm{g} / \mathrm{cm}^{3}$. Verificamos na Tabela 1, através da comparação de medidas diretas $\left(\mathrm{H}_{\mathrm{verd}}\right)$ com os resultados da inversão $\left(\mathrm{H}_{\text {calc }}\right)$, que os resultados para os modelos invertidos no trabalho foram satisfatórios.

Tabela 1. Comparação de medidas diretas $\left(\mathrm{H}_{\text {verd }}\right)$ com os resultados da inversão $\left(\mathrm{H}_{\text {calc }}\right)$ e $\Delta(\%)$ discrepância. Fonte: Dutra e Argollo (2017).

\begin{tabular}{ccccc}
\hline Longitude & Latitude & $\mathbf{H}_{\mathbf{v e r d}}$ & $\mathbf{H}_{\text {calc }}$ & $\Delta(\mathbf{\%})$ \\
\hline-36.81 & -10.60 & 2.44 & 2.36 & 5.26 \\
-36.79 & -10.57 & 3.00 & 2.95 & 1.65 \\
-36.06 & -10.00 & 1.00 & 0.95 & 4.41 \\
-36.04 & -9.99 & 1.11 & 1.17 & 4.97 \\
-35.92 & -9.68 & 3.19 & 3.24 & 1.53 \\
-35.91 & -9.59 & 2.05 & 2.12 & 3.56 \\
-35.89 & -9.59 & 2.66 & 2.57 & 3.23 \\
-35.80 & -9.59 & 3.12 & 3.00 & 3.99 \\
-35.75 & -9.55 & 3.00 & 3.08 & 1.50 \\
-35.71 & -9.58 & 3.47 & 3.53 & 5.43 \\
\hline
\end{tabular}

\section{CONSIDERAÇÕES FINAIS}

Neste trabalho foram apresentadas estimativas para parâmetros como espessura do embasamento e variação lateral do contraste de densidade nos domínios Estância, Canudos-VazaBarris, Macururé e Terreno Pernambuco-Alagoas no trecho adjacente à Bacia Sergipe-Alagoas.

Os modelos de contraste de densidade em profundidade obtidos a partir de dados gravimétricos residuais onde cada solução obtida possui um valor máximo de $\Delta \rho=0,25 \mathrm{~g} / \mathrm{cm}^{3}$. As principais estruturas regionais foram mapeadas em profundidade. Sugere-se a existência de intrusões de granitóides, com profundidades que variam de 10 a 15 km, ocorrendo no TPAL. Este terreno atuou de forma significativa nesta porção da Província Borborema e foi responsável pelo deslocamento para norte das massas de rochas do domínio Rio Coruripe sobre os metamorfitos e 
plutonitos do Domínio Pernambuco-Alagoas. A estrutura encontrada no domínio Rio Coruripe tem profundidade máxima de $10 \mathrm{~km}$. Esses resultados interferem na estrutura vertical do embasamento, e na produção de calor radiogênico gerado pelas rochas do embasamento e no fluxo de calor superficial total. Esse fluxo desempenha um papel relevante na história térmica da bacia, sendo seu conhecimento importante para avaliar a geração de hidrocarbonetos. Essa avaliação, realizada por meio da modelagem termomecânica da bacia, requer o conhecimento da massa específica e das propriedades térmicas das rochas do embasamento e sedimentos das bacias, além da geometria e profundidade das fontes.

A partir do conhecimento da anomalia gravimétrica e o contraste de densidade na superfície da bacia em superfície, foi possível delinear o relevo do embasamento da Bacia Sergipe-Alagoas. A área 1 apresentou valores máximos para espessura de 4,0 $\mathrm{km}$ de profundidade. A área 2 apresentou valores máximos para espessura de $3,5 \mathrm{~km}$ de profundidade com o relevo do embasamento apresentando mudanças ocasionalmente abrutas.

As anomalias gravimétricas foram invertidas com contraste de densidade de $\Delta \rho=-0,25$ $\mathrm{g} / \mathrm{cm}^{3}$. O método de inversão se mostrou eficaz para se obter o delineamento do relevo do embasamento e as espessuras apresentam correspondência com as anomalias observadas.

As bacias sedimentares representam a principal fonte de concentração de petróleo. O estudo dessas bacias pode revelar a existência de petróleo em acumulações comercialmente exploráveis. Porém, a acumulação do petróleo depende da existência de armadilhas estruturais que o aprisionem e promova sua concentração, somada à existência de matéria orgânica em quantidade, sua preservação e acumulação em profundidade. Por isso se torna importante apresentar resultados como o deste trabalho, que delineiam a interface entre o sedimento e embasamento da Bacia, e mapeiam estruturas do embasamento adjacente à bacia em profundidade.

\section{REFERÊNCIAS}

Aquino, G. S., Lana, M. C. 1990. Exploração na Bacia de Sergipe-Alagoas: o "estado da arte". Boletim de Geociências da Petrobrás, Rio de Janeiro, 4(1):75-84.

Barbosa, A G., 1990. Folha Limoeiro: Programa de Levantamentos Geológicos Básicos do Brasil. Recife: CPRM/DNPM. 124 p. 1:100.000.

Barbosa, V. C. F., and Silva, J. B. C., 1994, Generalized compact gravity inversion: Geophysics, 59, 57-68. 
Barbosa, V. C. F.; Silva, J. B. C.; Medeiros, W. E. 1997. Gravity inversion of basement relief using approximate equality constraints on depth. Geophysics, 62, 1745-1757.

Barbosa, V. C. F.; Silva, J. B. C.; Medeiros, W. E. 1999. Gravity inversion of a discontinuous relief stabilized by weighted smoothness constraints on depth. Geophysics, 64, 1429-1438.

Blakely, R.J., 1996, Potential Theory in Gravity and Magnetic Applications, Cambridge University Press, $441 \mathrm{pp}$.

Bhaskara, Rao, D. 1986. Modelling of sedimentary basins from gravity anomalies with variable density contrast. Geophys.J. Roy. Astr. Soc., 84: 207-121.

Brito Neves, B.B., Santos, E.J., Schmus, W.R.V., 2000. Tectonic history of the Borborema Province. In: Umberto Cordani; Edson Jose Milani; Antonio Thomaz Filho; Diogenes de Almeida Campos. (Org.). Tectonic Evolution of south america. Rio de Janeiro: 31st International Geological Congress, pp. 151e182. Sp. Publication.

Cainelli, C., Mohriak, W.U. 1998. Geology of Atlantic Eastern Brazilian basins. 1998. In: AAPG, International Conference \& Exhibition Short Course - Brazilian Geology Part II, Rio de Janeiro, Brazil, 67 p.

Chai, Y. \& Hinze, W. J. 1988. Gravity inversion of an interface above which the density contrast varies exponentially with depth. Geophysics, 53: 837-845.

Cordell, L. 1973. Gity analysis using an exponential density-depth function - San Jacinto Graben California. Geophysics, 38: 684-690.

Cruz, L. R. (2008) Caracterização Tectono-Estratigráfica da Sequiência Transicional na Sub- Bacia de Sergipe, Tese de Doutorado, Universidade Federal do Rio Grande do Norte.

Feijó, F. e Vieira, R. (1991) Sequências cretáceas das bacias de sergipe e alagoas., Geociências, 10(1):153-168.

Falkenhein, F.; Fujita, A.; Fernandes, G. et al. (1986) Análise da bacia de Sergipe-Alagoas, Petrobras. Relatório interno (não editado).

Guillen, A., and Menichetti, V., 1984, Gravity and magnetic inversion with minimization of a specific functional: Geophysics, 49, 1354-1360.

Hinze, William J., Ralph R. B. von Frese, and Afif H. Saad, 2013, Gravity and Magnetic Exploration: Principles, Practices, and Applications: Cambridge University Press.

Last, B. J., and Kubik, K., 1983, Compact gravity inversion: Geophysics, 48, 713-721. 
Litinsky, V. A. 1989. Concept of effective density: Key to gravity determinations for sedimentary basins. Geophysics, 54: 1474-1482.

Long, L. T. e Kaufmann, R. D., 2013. Acquisition and Analysis of Terrestrial Gravity Data. Cambridge University Press, 179 p., ISBN 978-1-107-02413-7.

Lowrie, W., 1997, Fundamentals of Geophysics, Cambridge University Press, 354 pp.

Mohriak, W.U. 1995. Elusivesal tectonics in the deep-water region of the Sergipe-Alagoas basin: evidence from deep seismic reflection profiles. In: SBGf, International Congress of the Brazilian Geophysical Society, 4, Rio de Janeiro, 51-54.

Mottaghya, D., Schellschmidtb,T. R., Popovc, Y. A., Clausera, C., Kukkonend, I.T., Novere ,G., Milanovskyf, S., Romushkevichc, R.A., 2005. New heat flow data from the immediate vicinity of the Kola supr-edeep borehole: Vertical variation in heat flow confirmed and attributed to advection. Tectonophysics, 401, $119-142$.

Murthy, I. V. R., \& Rao, D.B.. 1979. Gravity anomalies of two dimensional bodies of irregular cross section with density contrast varying with depth. Geophysics, 44 (9): 1525-1530.

Oliveira, R.G., 2008. Arcabouço Geofísico, Isostasia e causas do magmatismo cenozoco da Província Borborema e de sua Margem Continental (NE do Brasil) (TESE). Universidade Federal do Rio Grande do Norte, Natal, p. 411

Oliveira, E.P., Windley, B.F., Araújo, M.N.C., 2010. The Neoproterozoic Sergipano orogenic belt, NE Brazil: a complete plate tectonic cycle in western Gondwana. Precambrian Res. 181, 64-84.

Santos, E.J., Brito Neves, B.B., Van Schmus, W.R., Oliveira, R.G., Medeiros, V.C., 2000. An overall view on the displaced terrane arrangement of the Borborema Province, NE Brazil. In: International Geological Congress, 31th, Rio de Janeiro, Brazil, General Symposia, Tectonic Evolution of South American Platform, pp. 5-9.

Santos et al., 2014. Metalogênese das porções norte e central da Província Borborema, no livro "Metalogênese das Províncias Tectônicas Brasileiras. Editado pela CPRM. Organizadores: Maria da Glória da Silva, Manoel Barreto da Rocha Neto, Hardy Jost, Raul Minas Kuyumijan. P. 343-388.

Sari, Cokun \& Salk, Müjgan. 2002. Analysis of gravity anomalies with hyperbolic density contrast: An application to the gravity data of Western Anatolia. Journal of the Balk an Geophysical Society, 5 (3): 87-96. 
Silva Filho, A. F.; Guimaraes, I. P.; Brito, M. F. L.; Pimentel, M. M., 1997. Geochemical signatures of main Neoproterozoic Late-Tectonic granitoids from the Sergipano Fold Belt, Brazil: Significance For The Brasiliano Orogeny. International Geology Review, Estados Unidos, v. 39, n.07, p. 639659.

Silva, J. B. C., Medeiros, W. E., and Barbosa, V. C. F., 2000, Gravity inversion using convexity constraints. Geophysics, 65, 102-112.

\section{AGRADECIMENTOS}

Agradecemos ao Promob - Cenpes - Petrobras pelo apoio através do projeto Petrobras 0050.0082543.13-9.

Recebido em: 23/01/2017

Aceito para publicação em: 24/06/2017 